\title{
Multi-Scale Imaging of Tissues and Organoid Models by Backscattered Electron Scanning Microscopy after Pre- and Post-Embedding Immuno-Labeling
}

\author{
Mike Reichelt ${ }^{1 *}$, Danielle Mandikian ${ }^{2}$, Alejandro Lopez ${ }^{3}$, Anand K. Katakam ${ }^{1}$, Derek Smith ${ }^{4}$, William \\ Meilandt ${ }^{5}$, Jose Imperio ${ }^{5}$ and Meredith Sagolla ${ }^{1}$ \\ 1. Department of Pathology, Genentech, South San Francisco, USA. \\ 2. Department of PTPK, Genentech, South San Francisco, USA. \\ 3. Department of Biochemical and Cellular Pharmacology, Genentech, South San Francisco, USA. \\ 4. Department of Molecular Biology, Genentech, South San Francisco, USA. \\ 5. Department of Neuroscience, Genentech, South San Francisco, USA. \\ * Corresponding author: reichelm@gene.com
}

Ultrastructural investigation of tissues plays an important role in pathology, safety assessment and basic biomedical research. We and others have demonstrated that back-scattered electron scanning electron microscopy (BSE-SEM) can efficiently replace the more cumbersome traditional TEM approach for the ultrastructural analysis of tissue sections, because of its advantage of unobstructed imaging of large fields of tissues directly on histology glass slides over a wide range of magnification and resolution (multi-scale imaging). This saves significant "hands on" time and allows for the correlation of the ultrastructural detail with the histological context [1]. The benefits of this imaging method can be further increased by combining it with the power of immuno-labeling and fluorescence staining. Here we report on our efforts to combine various pre- and post-embedding labeling strategies with BSE-SEM imaging for projects in basic organoid-research and neuropathology.

Stem-cell derived kidney organoid models are used in research of kidney development and renal pathology as well as in studies of kidney toxicology and pharmacology [2]. While immunofluorescence microscopy (IF) can reveal the overall tissue organization, marker protein localization and regions of interest (ROIs), ultrastructural hallmarks of differentiated kidney-like organoids (basement membrane, podocyte foot-processes and brush-border microvilli) can only be resolved and visualized by electron microscopy (EM). We developed a strategy that combined fluorescent nuclear staining (Hoechst) with anti-Laminin IF-labeling and BSE-SEM to identify apoptotic cell nuclei, tubule-like tissue areas and laminin-expressing basement membrane-like structures (Figure 1A and 1B). We are currently working to further improve the ultrastructural preservation of the organoid samples and to identify and localize additional kidney marker proteins for further characterization of the kidney-organoid models.

In addition, we developed correlative IF and BSE-SEM strategies for the characterization of amyloid plaques in a PS2APP transgenic mouse model of Alzheimer's disease [3]. A pre-embedding immunogold-labeling protocol with an anti-amyloid antibody and silver enhancement of nanogold particles was established for the specific detection of amyloid-fibrils and amyloid-plaques in the hippocampus. Multi-scale BSE-SEM imaging was then used to find plaques by low magnification overview mapping of the hippocampal formation, followed by detection of individual gold particles enriched in amyloid-plaques by imaging at high magnification and resolution (Figure 1C). In a complimentary approach, we developed a post-embedding labeling protocol to detect amyloid-plaques by anti-amyloid IF-labeling with specific antibodies followed by combined IF and BSE-SEM imaging (Figure 1D). Amyloid-plaques could be visualized and quantified in overview fluorescence images. The 
higher resolution of BSE-SEM was then used to confirm the presence of amyloid-fibrils in the core of the amyloid-plaques (Figure 1D).

\section{References:}

[1] IU Wacker et al., J Vis Exp. 133 (2018). doi:10.3791/57059. PubMed PMID:29630046.

[2] M Takasato et al., Nature. 526 (2015), p. 564. doi:10.1038/nature15695.PMID:26444236.

[3] JG Richards et al., J Neurosci. 23 (2003), p. 8989. PubMed PMID:14523101.
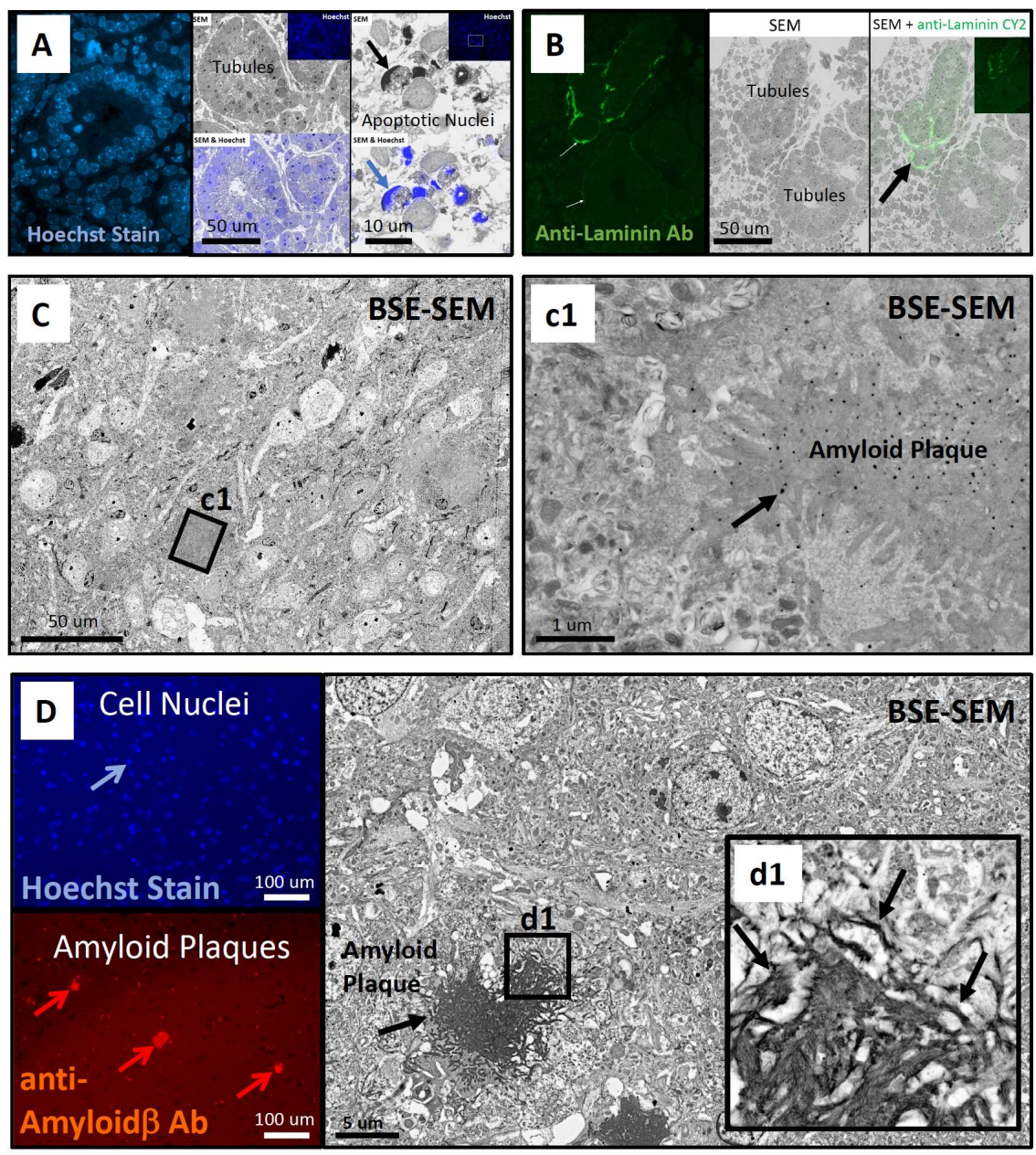

Figure 1. Examples of combined immuno-labeling and BSE-SEM imaging of organoid models (A) or brain slices (C and D). (A) Nuclear "Hoechst" staining (left) in iPSC-derived kidney organoids combined with BSE-SEM images of tubular organoid areas (middle) and apoptotic cell nuclei (right). (B) Anti-Laminin immunofluorescence staining of kidney organoids (left) combined with BSE-SEM images of tubular areas (middle and right). (C) Anti-amyloid pre-embedding labeling of amyloidplaques in the hippocampus of the PS2APP transgenic mouse model. Arrow depicts silver-enhanced gold particles (c1) enriched in the amyloid-plaque. (D) "Hoechst" nuclear staining (top left) and postembedding anti-amyloid immunofluorescence labeling (bottom left) to detect plaques in the PS2APP transgenic mouse model. BSE-SEM imaging revealed dense plaques with amyloid-fibrils (right, inset). 